\title{
Karyotype Reorganization with Conserved Genomic Compartmentalization in Dot-Shaped Microchromosomes in the Japanese Mountain Hawk-Eagle (Nisaetus nipalensis orientalis, Accipitridae)
}

\author{
C. Nishida ${ }^{a, b}$ J. Ishijima ${ }^{\text {b }}$ S. Ishishitac ${ }^{\text {K. Yamadab }}$ D.K. Griffin $^{\text {e }}$ T. Yamazaki ${ }^{d}$ \\ Y. Matsudac \\ ${ }^{\mathrm{a}}$ Department of Natural History Sciences, and ${ }^{\mathrm{b} C e n t e r ~ f o r ~ G e n o m e ~ D y n a m i c s, ~ F a c u l t y ~ o f ~ S c i e n c e, ~ H o k k a i d o ~ U n i v e r s i t y, ~}$ \\ Sapporo, 'Laboratory of Animal Genetics, Department of Applied Molecular Biosciences, Graduate School of \\ Bioagricultural Sciences, Nagoya University, Nagoya, and ${ }^{\mathrm{d}}$ The Project Team for Research and Conservation of the

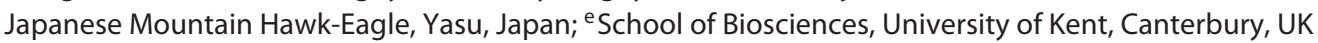

\section{Key Words}

Centromeric repetitive sequence - Chromosome homology . FISH · Japanese mountain hawk-eagle · Karyotype evolution · Microchromosome

\begin{abstract}
The karyotype of the Japanese mountain hawk-eagle (Nisaetus nipalensis orientalis) $(2 n=66)$ consists of a large number of medium-sized and small chromosomes but only 4 pairs of dot-shaped microchromosomes, in contrast to the typical avian karyotype with a small number of macrochromosomes and many indistinguishable microchromosomes. To investigate the drastic karyotype reorganization in this species, we performed a molecular cytogenetic characterization employing chromosome in situ hybridization and molecular cloning of centromeric heterochromatin. Cross-species chromosome painting with chicken chromosome-specific probes $1-9$ and $Z$ and a paint pool of 20 microchromosome pairs revealed that the $N$. n. orientalis karyotype differs from chicken by at least 13 fissions of macrochromosomes and 15 fusions between microchromosomes and between microand macrochromosomes. A novel family of satellite DNA sequences (NNO-Apal) was isolated, consisting of a GC-rich
\end{abstract}

173-bp repeated sequence element. The NNO-Apal sequence was localized to the C-positive centromeric heterochromatin of 4 pairs of microchromosomes, which evolved concertedly by homogenization between the microchromosomes. These results suggest that the 4 pairs of dot-shaped microchromosomes have retained their genomic compartmentalization from other middle-sized and small chromosomes.

(c) 2013 S. Karger AG, Basel

The typical avian karyotype has a high diploid chromosome number (mode, $2 \mathrm{n}=80$ ), consisting of a small number of large and medium-sized macrochromosomes and a large number of indistinguishable microchromosomes. This karyotype is widely conserved in all palaeognathous and most neognathous bird species [Christidis, 1990]. By contrast, atypical karyotypes characterized by a lower diploid number, no large macrochromosomes, many medium-sized and small chromosomes, and only a few pairs of microchromosomes are commonly found in Accipitridae (Falconiformes), including hawks, harriers, buzzards, kites, eagles, and Old World vultures [Takagi and Sasaki, 1974; de Boer, 1976; de Boer and Sinoo, 1984;

\section{KARGER}

E-Mail karger@karger.com www.karger.com/cgr
(C) 2013 S. Karger AG, Basel

$1424-8581 / 13 / 1414-0284 \$ 38.00 / 0$ 
Sasaki et al., 1984; Bed'Hom et al., 1998, 2003]. Karyotypes have been studied for 51 species in Accipitridae [reviewed by Amaral and Jorge, 2003], and the diploid chromosome number is $2 \mathrm{n}=66$ or 68 in most species.

Comparative gene mapping of the Chinese soft-shelled turtle (Pelodiscus sinensis) revealed that the 8 largest macrochromosomes were equivalent between the turtle $(2 \mathrm{n}=$ 66 ) and chicken ( $2 n=78$, typical karyotype) and that the turtle microchromosomes also correspond to chicken microchromosomes and chromosome $4 \mathrm{p}$ [Matsuda et al., 2005; Uno et al., 2012], suggesting that this reptile and birds share the same ancestral karyotype. Chromosome painting studies with chicken chromosome-specific probes have been performed in 52 avian species representing 12 orders. All macrochromosomes are highly conserved in 10 orders because only some structural changes have occurred in macrochromosomes [Shetty et al., 1999; Raudsepp et al., 2002; Guttenbach et al., 2003; Kasai et al., 2003; Derjusheva et al., 2004; International Chicken Genome Sequencing Consortium, 2004; Shibusawa et al., 2004a, b; Itoh and Arnold, 2005; NishidaUmehara et al., 2007; de Oliveira et al., 2008; Nie et al., 2009; Nanda et al., 2011]. However, an increase in the diploid number by microchromosome fissions or a drastic decrease in chromosome number by extensive microchromosome fusions have been found in several species (double-wattled cassowary, $2 \mathrm{n}=92$ [Nishida-Umehara et al., 2007]; common coot, $2 \mathrm{n}=92$ [Nanda et al., 2011]; stone curlew, $2 \mathrm{n}=42$ [Nie et al., 2009]). In the remaining orders Falconiformes [de Oliveira et al., 2005, 2010; Nanda et al., 2006; Nishida et al., 2008] and Psittaciformes [Nanda et al., 2007], drastic rearrangements of macrochromosomes have occurred. Chromosome homology has been analyzed between chicken and 5 accipitrid species: the harpy eagle (Harpia harpyja) [de Oliveira et al., 2005], 3 Old World vultures (Gyps rueppelli, G. fulvus, Gypaetus barbatus) [Nanda et al., 2006] and the white hawk (Leucopternis albicollis) [de Oliveira et al., 2010]. These studies revealed that 2 major events of drastic chromosomal reorganization have occurred during karyotype evolution in these species: fissions of large macrochromosomes and fusions between microchromosomes and between micro- and macrochromosomes.

Centromeric repetitive DNA sequences, which are associated with centromeric heterochromatin and represent long tandem arrays, have been isolated from many higher vertebrates [Kalitsis and Choo, 1997]. Satellite DNA sequences, a major component of highly repetitive DNA in the eukaryotic genome, provide good taxonomic and phylogenetic markers for reconstructing evolutionary rela-

Karyotype Reorganization in the Mountain Hawk-Eagle tionships between closely related species that share the same repeated sequence family [Yamada et al., 2004]. Centromeric repetitive DNA sequences have been molecularly cloned and characterized in several avian species. Microchromosome-specific centromeric repetitive DNA sequences have been found in 4 galliform species (chicken, Gallus gallus; turkey, Meleagris gallopavo; Japanese quail, Coturnix japonica; blue-breasted quail, C. chinensis) and 2 struthioniform species (lesser rhea, Pterocnemia pennata; greater rhea, Rhea americana) [Matzke et al., 1990, 1992; Tanaka et al., 2000; Yamada et al., 2002a, b], all of which have the typical avian karyotype with centromeric heterochromatin in both macro- and microchromosomes, indicating chromosome size-dependent genomic compartmentalization between macro- and microchromosomes. However, at the onset of our study, nothing was known about centromeric repetitive sequences and their chromosomal distribution in accipitrids with no large macrochromosomes and few microchromosomes.

The mountain hawk-eagle (Nisaetus nipalensis; synonym Spizaetus nipalensis; Accipitridae, Falconiformes) has an atypical karyotype with $2 \mathrm{n}=68$, consisting of 60 medium-sized and small chromosomes including the $\mathrm{Z}$ and $\mathrm{W}$ sex chromosomes and only 8 minute elements [Takagi and Sasaki, 1974]; however, little is known about the process by which the drastic changes in chromosomal organization occurred in this species. To understand how the genome organization consisting of macro- and microchromosomes has been modified in Accipitridae, we used cross-species hybridization with chicken chromosomespecific DNA probes to characterize N. nipalensis orientalis at the molecular cytogenetic level. We also did chromosome mapping of the MHC and 18S-28S ribosomal RNA (rRNA) genes and telomeric (TTAGGG)n sequences, examined chromosome homologies with chicken and delineated the pattern of drastic genomic reorganization in this species. Finally, to examine the correlation between karyotype reorganization and the chromosomal distribution of centromeric repetitive sequences, we molecularly cloned centromeric repetitive DNA sequences and characterized them by nucleotide sequencing, chromosome in situ hybridization and filter hybridization.

\section{Materials and Methods}

Cell Culture and Chromosome Preparation

For cell culture, a small piece of skin tissue was collected from each of 7 female and 1 male Japanese mountain hawk-eagles (N. $n$. orientalis), which had been captured for another research study and were released after biopsy. Fibroblast cells were cultured at 
$39^{\circ} \mathrm{C}$ in $5 \% \mathrm{CO}_{2}$ in Medium 199 supplemented with $18 \%$ fetal bovine serum. The cells were collected after $15 \mathrm{~min}$ of colcemid $(0.025 \mu \mathrm{g} / \mathrm{ml})$ treatment, suspended in $0.075 \mathrm{M} \mathrm{KCl}$ for $20 \mathrm{~min}$ at room temperature and fixed with 3:1 methanol/acetic acid. Chromosome preparations for Giemsa-stained karyotype analysis and C-banding were made following the standard air-drying method. Replication-banded chromosome slides were made for in situ hybridization as described previously [Matsuda and Chapman, 1995; Nishida-Umehara et al., 2007]. 5-Bromodeoxyuridine $(25 \mu \mathrm{g} / \mathrm{ml})$ was added to the culture medium, and the cells were cultured for an additional $5 \mathrm{~h}$, including $15 \mathrm{~min}$ of colcemid $(0.025 \mu \mathrm{g} / \mathrm{ml})$ treatment before harvesting. The chromosome slides were stained with Hoechst $33258(1 \mu \mathrm{g} / \mathrm{ml})$ for $5 \mathrm{~min}$, heated to $65^{\circ} \mathrm{C}$ for $3 \mathrm{~min}$ on a hot plate, exposed to UV light at $65^{\circ} \mathrm{C}$ for $5 \mathrm{~min}$, and finally stored at $-80^{\circ} \mathrm{C}$ until use.

\section{C-Banding}

To examine the chromosomal distribution of centromeric heterochromatin, C-banding was performed with the barium hydroxide/saline/Giemsa method [Sumner, 1972] with slight modification; chromosome slides were treated with $0.2 \mathrm{M} \mathrm{HCl}$ for $5 \mathrm{~min}$ at room temperature and then with $5 \% \mathrm{Ba}(\mathrm{OH})_{2}$ at $50^{\circ} \mathrm{C}$ for $1-2 \mathrm{~min}$.

\section{Chromosome Painting}

Cross-species chromosome painting with chicken probes was performed as described previously [Nishida-Umehara et al., 2007]. Chicken (G. gallus, GGA) chromosome-specific DNA probes for chromosomes 1-9 and Z (GGA1-9 and GGAZ) [Griffin et al., 1999; Masabanda et al., 2004] and a mixture of microchromosome-specific paints, consisting of 20 pairs of chicken microchromosomes [Habermann et al., 2001; de Oliveira et al., 2005], were used. FISH images were photographed with Kodak Ektachrome ASA 100 film through a Nikon E800 fluorescence microscope.

\section{Comparative Mapping of the MHC and 18S-28S rRNA Genes} and of Telomeric Repeats and AgNOR Staining

Chromosomal localizations of the MHC and 18S-28S rRNA genes and telomeric repeats were performed using FISH as described in Matsuda and Chapman [1995]. The 90-kb BAC clone bw065G09 containing the MHC genes on chicken chromosome 16, provided by Dr. Crooijmans of Wageningen Agricultural University, the Netherlands [Crooijmans et al., 2000], was used. The 5.8-kb pHr21 Ab and 7.3-kb pHr14E3 fragments of the human ribosomal RNA genes were provided by the Japanese Cancer Research Resource Bank, Ibaraki, Japan. A biotin-labeled 42-bp oligonucleotide probe complementary to (TTAGGG)n sequences was used for chromosomal mapping of telomeric sequences. The DNA probes were labeled with biotin-16-dUTP (Roche Diagnostics, Basel, Switzerland) by nick translation following a standard protocol and ethanol-precipitated with salmon sperm DNA and $E$. coli tRNA. After hybridization, the probes were stained with avidin-FITC (Roche Diagnostics). For dual-color FISH, DNA probes were separately labeled with digoxigenin (DIG)-11-dUTP (Roche Diagnostics) and biotin-16-dUTP and stained with anti-DIG-rhodamine (Roche Diagnostics) and avidin-FITC, respectively. The chromosomal location of nucleolus organizer regions (NORs) was examined with the same metaphase spreads used for FISH analysis. After FISH, the slides were washed with 3:1 methanol/acetic acid for $5 \mathrm{~min}$, rinsed in distilled water and air-dried. AgNOR staining was performed as described in Howell and Black [1980].

\section{Molecular Cloning of Repetitive Sequences}

Genomic DNA was extracted from the blood of one female $N$. n. orientalis in the Asahiyama Zoo, Asahikawa, Hokkaido, Japan, following a standard protocol. Genomic DNA was digested with 23 restriction endonucleases (ApaI, BamHI, BglI, BglII, BstXI, DraI, EcoRI, EcoRV, HaeIII, HindIII, HinfI, MboI, MluI, NsiI, PstI, PvuII, RsaI, SacI, SalI, SmaI, TaqI, XbaI, XhoI), size-fractionated with $3 \%$ agarose gel electrophoresis and then stained with ethidium bromide. Prominent DNA bands of repeated sequences thus detected were eluted from the gel by using a QIAquick Gel Extraction Kit (Qiagen, Hilden, Germany), ligated into pBluescript II SK (+) and transformed into E. coli JM109 competent cells (Takara Bio, Otsu, Japan). The sizes of DNA fragments inserted in the vector were determined by electrophoresis of the PCR products amplified with T3 and T7 primers, and the clones were used for nucleotide sequencing and FISH analysis. Nucleotide sequences were determined with an ABI PRISM 3100 DNA Analyzer (Applied Biosystems, Foster, Calif., USA) after sequencing reactions with a Big Dye Terminator v1.1 Cycle Sequencing Kit (Applied Biosystems).

\section{Southern Blot Hybridization}

Genomic DNA from N. n. orientalis was digested with 5 restriction endonucleases (ApaI, HaeIII, HinfI, HpaII, MspI). The digested DNA was fractionated by electrophoresis in a horizontal $2 \%$ agarose gel and transferred onto a nylon membrane (Roche Diagnostics). Southern blot hybridization was performed as described previously [Yamada et al., 2006].

\section{Slot-Blot Hybridization}

Slot-blot hybridization was performed to examine the nucleotide sequence conservation among repetitive sequences from various species and to estimate the amount of repetitive sequence in the N. $n$. orientalis genome. Genomic DNA was collected from female individuals of 20 species from 7 orders, as follows: (1) Falconiformes: Japanese mountain hawk-eagle ( $N$. n. orientalis), northern goshawk (Accipiter gentilis) and crested serpent eagle (Spilornis cheela) (Accipitridae); osprey (Pandion haliaetus) (Pandionidae); peregrine falcon (Falco peregrinus) (Falconidae); Andean condor (Vultur gryphus) (Cathartidae). (2) Strigiformes: Blakiston's fish-owl (Bubo blakistoni; synonym Ketupa blakistoni) and Eurasian eagle owl (B. bubo) (Strigidae). (3) Gruiformes: Siberian white crane (Grus leucogeranus) and Stanley crane (G. paradisea; synonym, Anthropoides paradisea) (Gruidae); common trumpeter (Psophia crepitans) (Psophiidae) and common coot (Fulica atra) (Rallidae). (4) Ciconiiformes: black-faced spoonbill (Platalea minor) and Japanese crested ibis (Nipponia nippon) (Threskiornithidae). (5) Passeriformes: tree sparrow (Passer montanus saturatus) (Passeridae) and barn swallow (Hirundo rustica) (Hirundinidae). (6) Galliformes: chicken (G. gallus) (Phasianidae) and guinea fowl (Numida meleagris) (Numididae). (7) Struthioniformes: ostrich (Struthio camelus) (Struthionidae) and emu (Dromaius novaehollandiae) (Casuariidae).

Genomic DNA was denatured with $0.4 \mathrm{M} \mathrm{NaOH}$ for $10 \mathrm{~min}$ and transferred onto a nylon membrane (Roche Diagnostics) by using a BIO-DOT SF blotting apparatus (Bio-Rad, Hercules, Calif., USA). DNA probes were labeled with DIG-11-dUTP by using PCR DIG Labeling Mix and hybridized to the membrane at $42^{\circ} \mathrm{C}$ in DIG Easy Hyb solution. Chemiluminescent signals were detected with the same protocol used for Southern blot hybridization. 


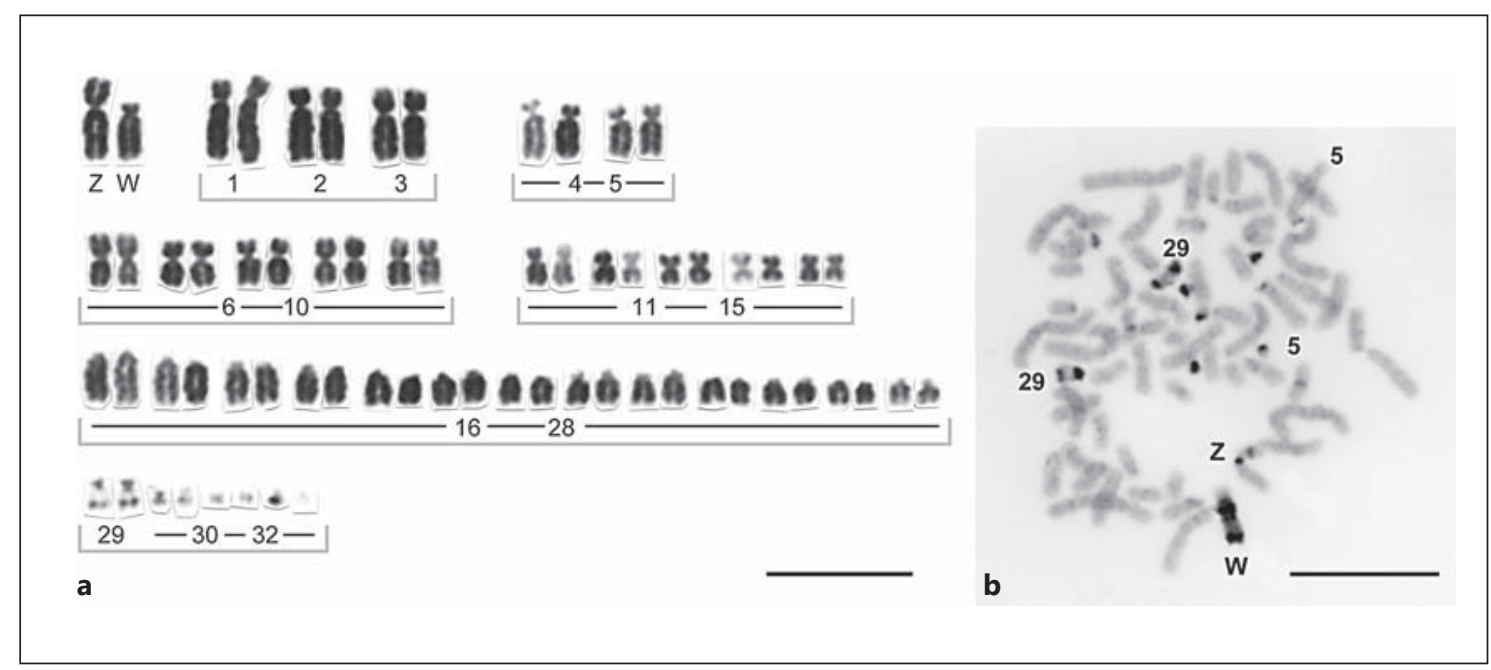

Fig. 1. a Giemsa-stained karyotype and b C-banded metaphase spread from a Japanese mountain hawk-eagle ( $N$. $n$. orientalis). Chromosomes are arranged in the karyotype by size and morphology and are classified into 6 groups of autosomes $(1-3,4-5,6-10,11-15,16-28,29-32)$ and the ZW sex chromosomes. Scale bars $=10 \mu \mathrm{m}$.

For estimating the amount of repetitive sequences in the genome of $N$. $n$. orientalis, 5 different concentrations of female genomic DNA and PCR products for the repeated sequence element were blotted on a membrane and used for hybridization. The intensity of hybridization bands was estimated and compared by using public-domain image processing and analysis software NIH Image (http://rsbweb.nih.gov/nih-image/).

\section{Results}

Karyotype of N. n. orientalis

The diploid chromosome number was 66 for all 8 individuals of $N$. $n$. orientalis. Although the size difference among chromosomes was sequential, the autosomes could be classified into 6 groups on the basis of size and morphology (fig. 1a): 3 pairs of large submetacentrics (13), 2 pairs of medium-sized subtelocentrics $(4,5), 5$ pairs of medium-sized meta- or submetacentrics (6-10), 5 pairs of small meta- or submetacentrics (11-15), 13 pairs of medium-sized and/or small acrocentrics (16-28), and 4 pairs of dot-shaped microchromosomes (29-32), of which chromosome 29 had a large secondary constriction. The submetacentric $\mathrm{Z}$ chromosome was of the same size category as chromosomes $1-3$, whereas the $\mathrm{W}$ chromosome was a medium-sized subtelocentric chromosome. Intensely C-positive, darkly staining heterochromatin was observed (fig. 1b) in the centromeric region of 4 pairs of microchromosomes (29-32) and the Z chromosome, in the long arm of chromosome 29 except for the secondary constriction and in the long arm of $\mathrm{W}$ except for the interstitial region. Weak C-bands were observed at the distal end of the short arm of chromosome 5 , in the centromeric regions of several pairs in chromosome subgroups $6-10$ or $11-15$ and in the pericentromeric region of the $\mathrm{Z}$ long arm.

\section{Homology with Chicken Chromosomes}

Chromosome-specific paints for chicken (G. gallus) chromosomes 1-9 and Z (GGA1-9 and GGAZ) and a mixture of microchromosome-specific paints all efficiently cross-hybridized to metaphase chromosomes of $N$. n. orientalis (NNO) (fig. 2). Hoechst-stained bands obtained by replication banding, which correspond to Gbands, made it possible to identify most chromosomes except for 23-28 and 30-32. Homologies with GGA1-9 and $Z$ were found for 23 chromosome pairs, including the ZW sex chromosomes (fig. 3). GGA1 showed homology with 7 NNOs (NNO6, 13, 15, 19, 21, 2 pairs of 23-28), GGA2 with 3 chromosomes and/or chromosome segments (NNO2q, 3, 22 except for the distal part), GGA3 with 4 (NNO10 except for the q distal part, 12q, 17q, 20), GGA4 with 2 (NNO1, 14), and GGA5 with 2 (NNO11q, 16). GGA6, 7, 8, and 9 each hybridized to a single chromosome arm (NNO4q, 5q, 8q, and 9q, respectively). GGAZ hybridized to the entire $\mathrm{Z}$ chromosome and also showed cross-hybridization to the short arm and the interstitial region of the long arm of the $\mathrm{W}$ chromosome, which corresponded to the $\mathrm{C}$-negative regions. The mi- 
Fig. 2. Chromosome painting with chicken chromosome-specific DNA probes to PIstained metaphase chromosome spreads of N. n. orientalis. a The GGA1 probe hybridized to NNO6, 13, 15, 19, 21, and 2 pairs of 23-28. b The GGA3 probe hybridized to NNO10 (except for the distal part of the $\mathrm{q}$ arm), 12q, 17q, and 20. d GGAZ painted the $\mathrm{Z}$ chromosome and part of the $\mathrm{W}$ chromosome. e A paint pool of 20 chicken microchromosomes hybridized to 10 pairs of chromosomes, partially or entirely. The Hoechst-banded patterns of the PI-stained metaphase spreads in $\mathbf{b}$ and $\mathbf{e}$ are shown in c and f, respectively. Arrowheads indicate microchromosomes. Scale bars $=10 \mu \mathrm{m}$.
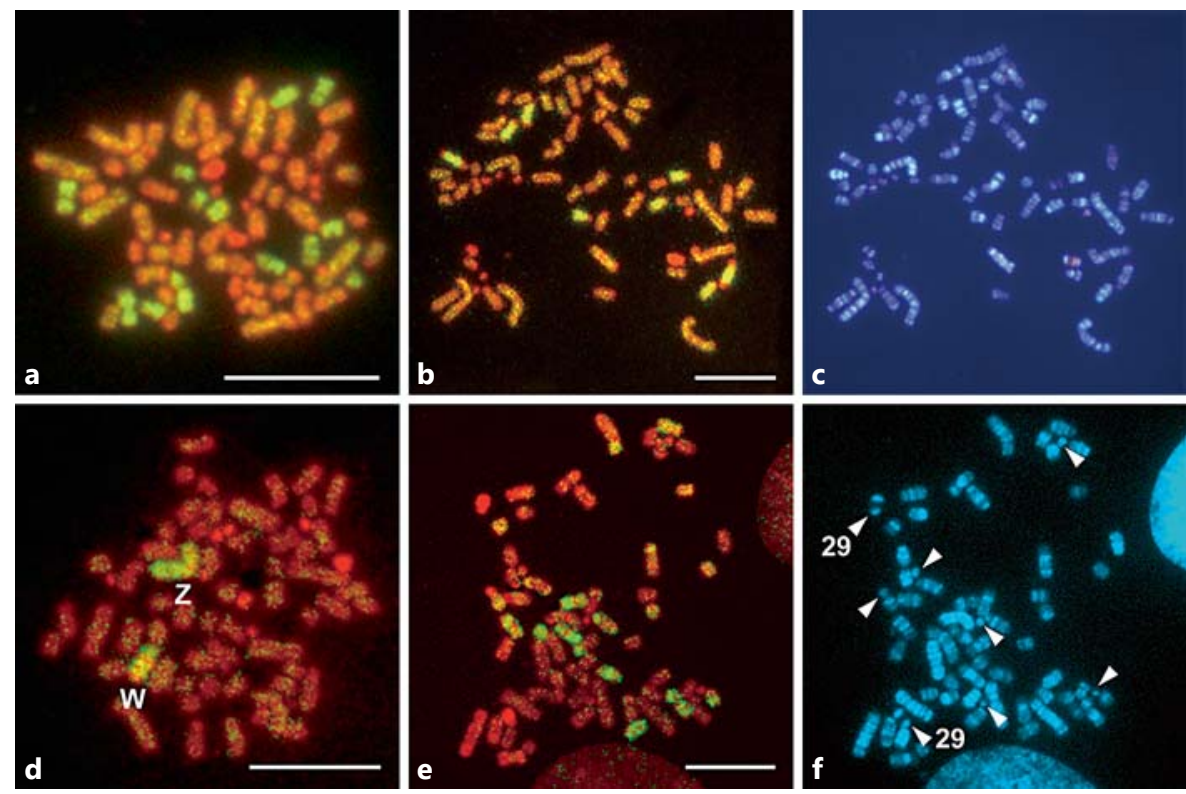

Fig. 3. Hoechst-banded karyotype of $N$. $n$. orientalis showing the assignment of homology with chicken chromosomes, established by chromosome painting with chicken probes. The homologous chicken chromosome is shown to the right of each set of $N$. n. orientalis chromosomes. At the lower right is the key to the color coding for chicken chromosomes 1-9 and Z and 20 microchromosome pairs (m).

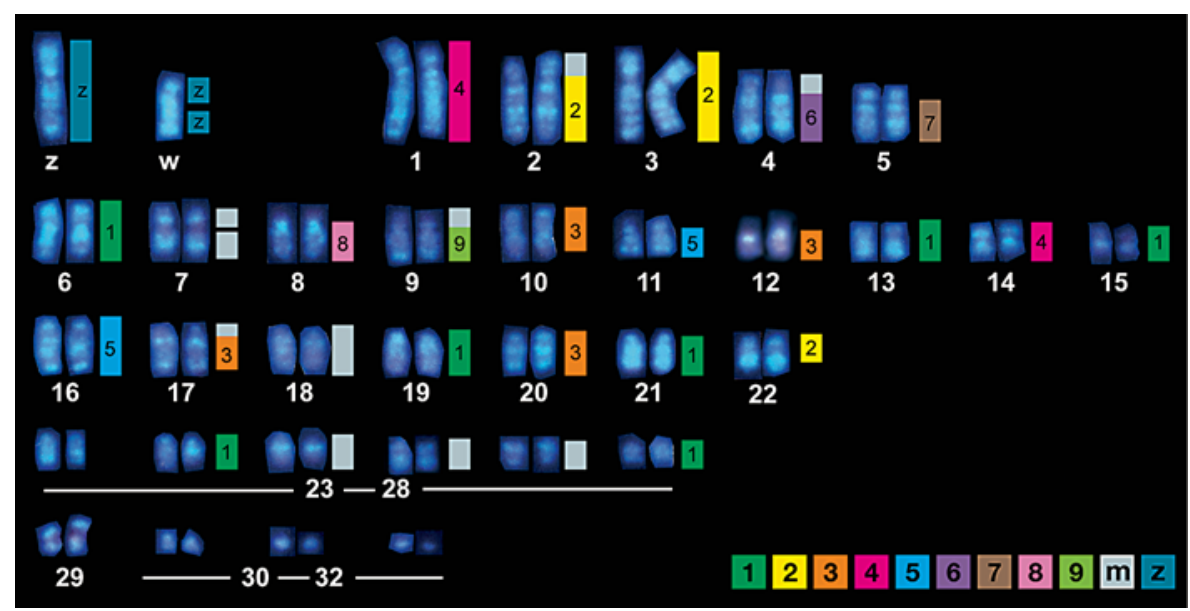

crochromosome-specific paint pool hybridized to at least 10 different chromosomes and/or chromosome segments: NNO2p, 4p, 7p, 7q, 9p, the pericentromeric region of 17,18 , and 3 pairs among 23-28 (fig. 3). No hybridization signals were detected with this paint pool for 4 pairs of dot-shaped microchromosomes (fig. 2e, f).

Chromosomal Distribution of the MHC and 18S-28S rRNA Genes and of Telomeric (TTAGGG)n Sequences and AgNOR Staining

The AgNOR-stained region (18S-28S rRNA genes) and the MHC genes were separately localized to the secondary constriction of microchromosome 29 and another pair of microchromosomes, respectively (fig. $4 \mathrm{a}-\mathrm{c}$ ), in contrast to the chicken, where these genes are located close together on the same microchromosome pair (chromosome 16) (fig. 4d, e). Telomeric (TTAGGG)n repeats were localized to both telomeric ends of all chromosomes, but no interstitial hybridization signals were detected (fig. 4f).

\section{Chromosomal Distribution and Nucleotide Sequences} of Repetitive Sequences

A prominent, approximately 170-bp band of repeated DNA sequences was detected by agarose gel electropho- 
Fig. 4. Chromosomal localization of NORs and of the MHC and 18S-28S rRNA genes in $N$. $n$. orientalis and chicken and chromosomal localization of telomeric (TTAGGG)n sequences in $N$. $n$. orientalis. a AgNOR staining pattern and $\mathbf{b}$ FISH pattern for the MHC genes on the same metaphase spread of $N$. $n$. orientalis. c FISH pattern for the 18S-28S rRNA genes on a metaphase spread of N. n. orientalis. d AgNOR staining pattern and e dual-color FISH pattern for the DIG-labeled MHC genes (red) and biotin-labeled 18S-28S rRNA genes (green) on the same metaphase spread of chicken. $\mathbf{f}$ FISH pattern for telomeric (TTAGGG)n sequences in $N$. $n$. orientalis. Scale bars $=10 \mu \mathrm{m}$.
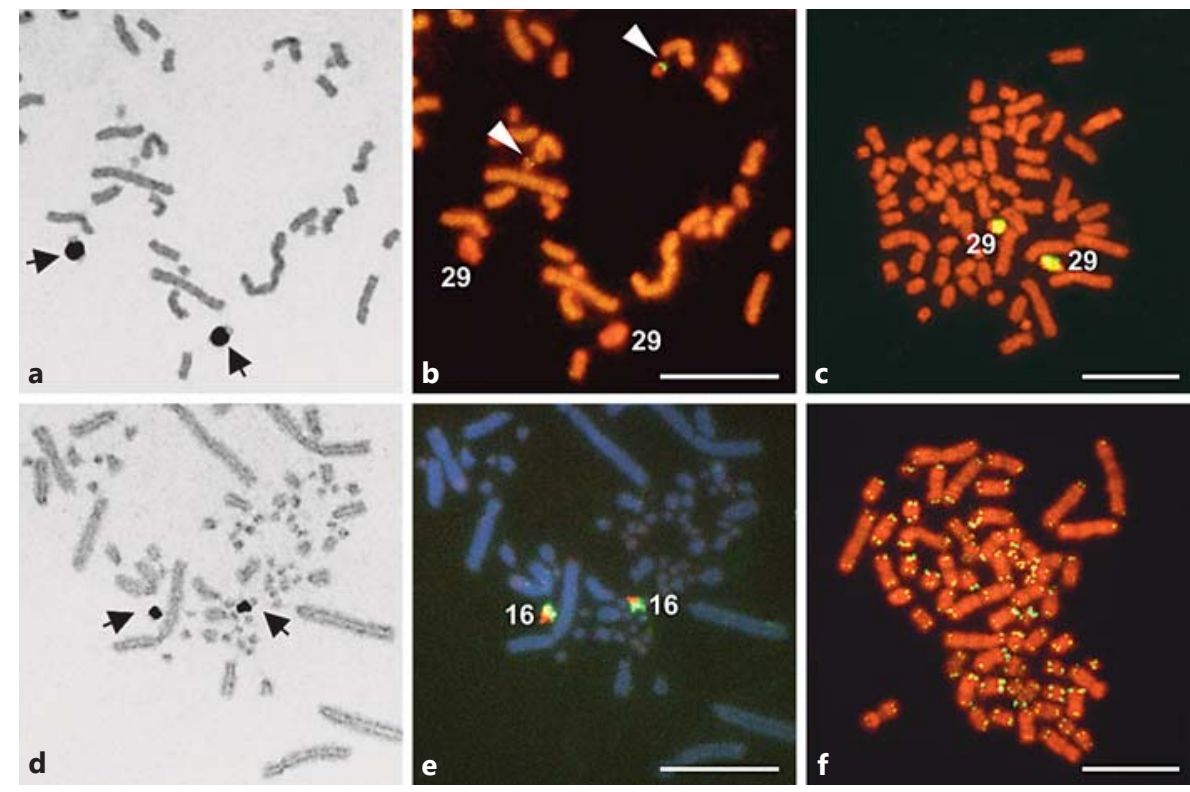
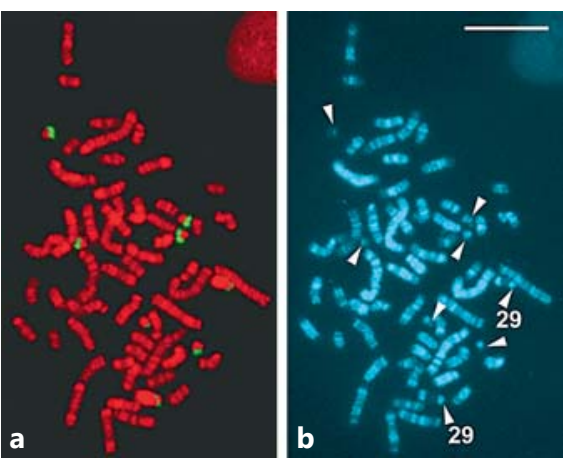

Fig. 5. Chromosomal distribution of a the NNO-ApaI-S1 fragment on a PI-stained metaphase chromosome spread of $N$. $n$. orientalis and $\mathbf{b}$ Hoechst-stained pattern of the same metaphase spread. Arrowheads indicate microchromosomes. Scale bar $=10 \mu \mathrm{m}$.

resis of ApaI-digested genomic DNA (online suppl. fig. 1; see www.karger.com/doi/10.1159/000352067 for all online supplementary material). The band was eluted from the gel, and DNA fragments were cloned into pBluescript SK $( \pm)$. Twenty-two clones were obtained, and their nucleotide sequences were determined. Fourteen of the 22 clones grouped in the same family of repetitive DNA sequences. The NNO-ApaI-S1 fragment of this repeated sequence family produced strong hybridization signals in the centromeric regions of 4 pairs of dot-shaped micro-

Karyotype Reorganization in the Mountain Hawk-Eagle chromosomes; the chromosomal distribution of these signals corresponded to centromeric C-bands (fig. 5). The length of 14 NNO-ApaI-S fragments (AB675097675110) was 172 or 173 bp (fig. 6). The average nucleotide sequence identity among the fragments was $92.0 \%$ (range, $86.7-98.3 \%$ ), and the average GC content was $57.4 \%$ (54.9-60.7\%). A similarity search of the DNA Data Base of Japan (http://www.ddbj.nig.ac.jp) detected high sequence identity between our fragments and a microsatellite DNA sequence $(96 / 100,96 \%)$ from a closely related species, Bonelli's eagle (Hieraaetus fasciatus).

\section{Organization of Repetitive Sequences in the Genome}

Hybridization bands for the NNO-ApaI element were observed as polymeric ladders of tandem repeats of the 173-bp monomer unit in the ApaI digest (fig. 7). In the HaeIII digest, a high-intensity hybridization band was found for the monomer unit, but no higher-order hybridization bands than the dimeric fragments were found, indicating that HaeIII restriction sites are more highly conserved than ApaI sites in the NNO-ApaI sequences. By contrast, HinfI sites were far fewer than the ApaI and HaeIII sites. The restriction site for both HpaII and MspI is CCGG. HpaII does not cleave, when the second cytosine is methylated, whereas MspI does. In the MspI digest, intense hybridization bands were observed at lower molecular weight with progressively decreasing copy number for each higher order of repeat units; by contrast, the 
Fig. 6. Aligned nucleotide sequences of 14 NNO-ApaI-S fragments. Dots indicate identity with nucleotides in the consensus sequence at the top, and hyphens indicate gaps. Parts of the ApaI site at both ends are outlined. Restriction sites for the other 3 endonucleases are indicated as follows:

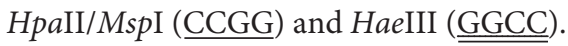

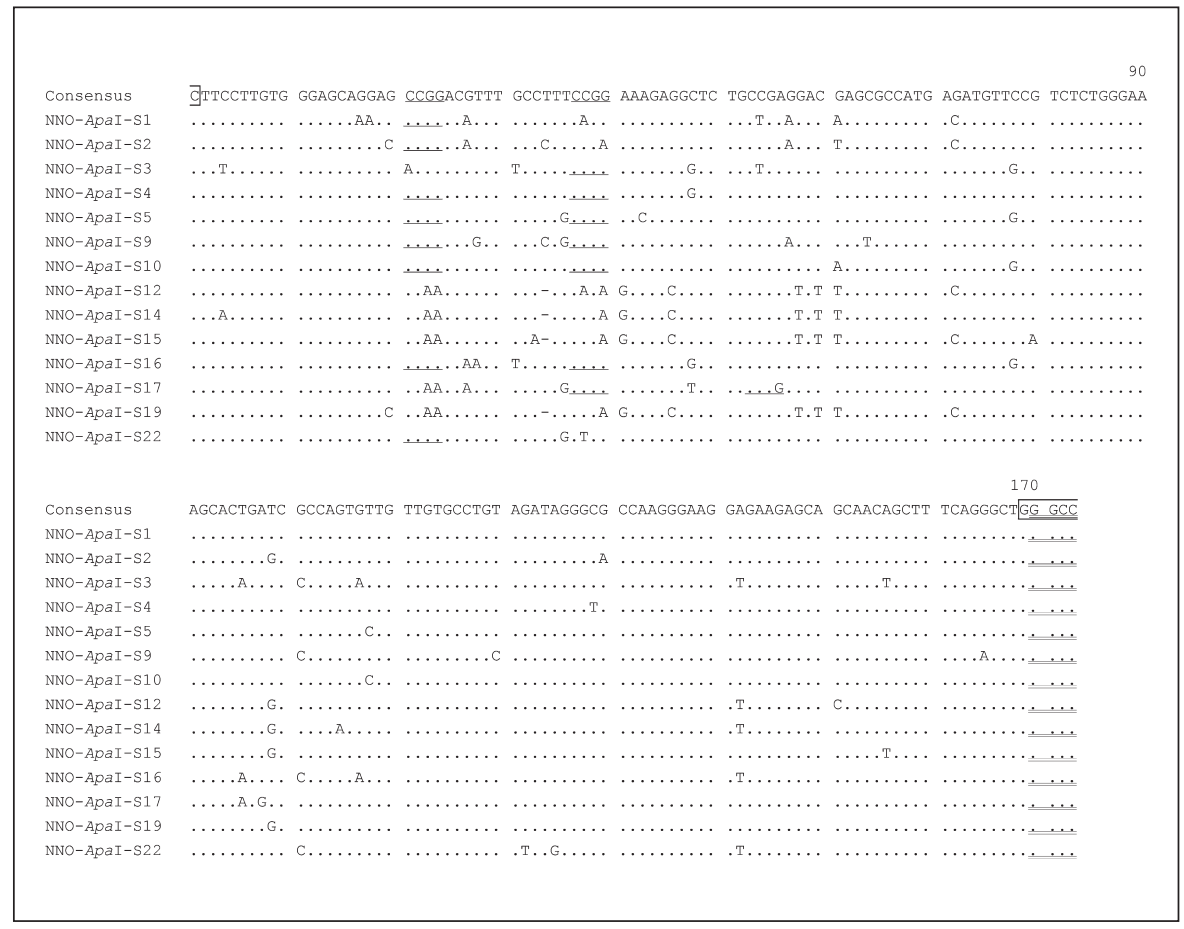

intensity of ladder bands increased from low to high molecular weight in the HpaII digest, indicating that the $\mathrm{NNO}-A p a \mathrm{I}$ repeated sequences were hypermethylated in blood cells.

The copy number of the NNO-ApaI sequence was measured by hybridizing the labeled NNO-ApaI fragment to genomic DNA and probe DNA using slot-blot hybridization (online suppl. fig. 2). The 173-bp NNOApaI element accounted for $0.48 \%\left(28.1 \times 10^{3}\right.$ copies/ haploid genome) of the N. n. orientalis genome.

\section{Conservation of the NNO-ApaI Repetitive Sequence}

Nucleotide conservation of the NNO-ApaI sequence was examined by slot-blot hybridization for 20 species from 7 orders (fig. 8). In Falconiformes, intense hybridization signals were found in Accipitridae (A. gentilis, $S$. cheela), Pandionidae (P. haliaetus) and Cathartidae (V. gryphus), but not in Falconidae (F. peregrinus). Hybridization signals were also observed in Gruiformes as an intense hybridization band for G. leucogeranus (Gruidae) and faint bands for G. paradisea (Gruidae) and P. crepitans (Psophiidae), but there was no band for F. atra (Rallidae). No hybridization signals were found for the other orders (Strigiformes, Ciconiiformes, Passeriformes, Galliformes, Struthioniformes).

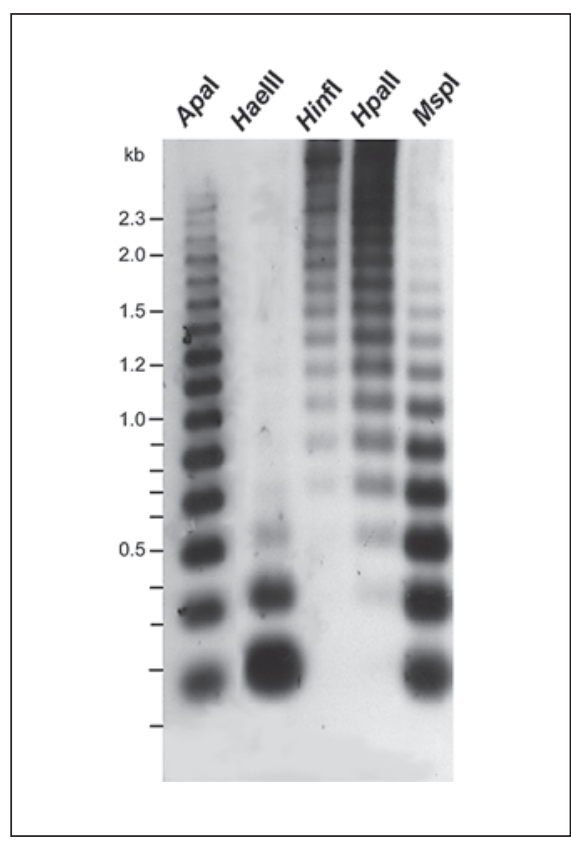

Fig. 7. Southern blot of N. n. orientalis genomic DNA digested with 5 endonucleases (ApaI, HaeIII, HinfI, HpaII, MspI), probed by hybridization with the NNO-ApaI-S fragment. 


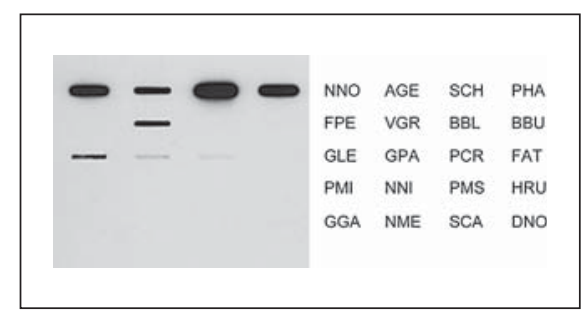

Fig. 8. Slot-blot probed with the NNO-ApaI-S fragment labeled with DIG-11-dUTP. Genomic DNA from 20 species representing the following 7 orders was blotted. (1) Falconiformes: NNO (N. $n$. orientalis), AGE (Accipiter gentilis), SCH (Spilornis cheela) (Accipitridae); PHA (Pandion haliaetus) (Pandionidae); FPE (Falco peregrinus) (Falconidae); VGR (Vultur gryphus) (Cathartidae). (2) Strigiformes: BBL (Bubo blakistoni), BBU (B. bubo) (Strigidae). (3) Gruiformes: GLE (Grus leucogeranus), GPA (G. paradisea) (Gruidae); PCR (Psophia crepitans) (Psophiidae); FAT (Fulica atra) (Rallidae). (4) Ciconiiformes: PMI (Platalea minor), NNI (Nipponia nippon) (Threskiornithidae). (5) Passeriformes: PMS (Passer montanus saturatus) (Passeridae); HRU (Hirundo rustica) (Hirundinidae). (6) Galliformes: GGA (Gallus gallus) (Phasianidae); NME (Numida meleagris) (Numididae). (7) Struthioniformes: SCA (Struthio camelus) (Struthionidae); DNO (Dromaius novaehollandiae) (Casuariidae).

\section{Discussion}

The diploid chromosome number of the Japanese mountain hawk-eagle (N. n. orientalis) was 66 for all 8 individuals examined in this study, and the $\mathrm{Z}$ and $\mathrm{W}$ sex chromosomes were submetacentric and subtelocentric, respectively. This result differed from that of Takagi and Sasaki [1974] in which the diploid chromosome number of an unknown subspecies of $N$. nipalensis was 68 , and the W was telocentric. The DNA Data Bank of Japan lists 4 subspecies for N. nipalensis (N. n. orientalis, N. n. nipalensis, N. n. taiwanensis, N. n. fokiensis), and the difference in chromosome number and $\mathrm{W}$ chromosome morphology between our study and the previous report might have resulted from different subspecies having been used for karyotype analysis.

Cross-species hybridization of $N$. $n$. orientalis chromosomes with paints for 10 chicken macrochromosomes (GGA1-9, Z) and a paint pool for 20 chicken microchromosomes detected 23 and 10 homologous chromosome regions, respectively, on 28 different chromosomes, indicating that $N$. $n$. orientalis differs from chicken by at least 13 fissions of macrochromosomes and 15 fusions of microchromosomes. The euchromatic short arm and Cnegative interstitial region of the $\mathrm{W}$ long arm were painted with the chicken $\mathrm{Z}$ probe. This homology between the
$\mathrm{Z}$ and $\mathrm{W}$ chromosomes has been also reported in the harpy eagle [de Oliveira et al., 2005] and common buzzard [Nanda et al., 2008], indicating that the sex chromosomes have not completely differentiated in Accipitridae in contrast to the majority of non-ratite birds.

In all 6 accipitrid species, including $N$. n. orientalis, chromosomal fragmentation by fission occurred only in the homologs of chicken chromosomes 1-5, whereas chicken chromosomes 6-9 are conserved. The numbers of chromosome pairs that hybridized with each paint GGA1-5 were, respectively: 5, 2, 4, 2, and 2 for $H$. harpyja (10 fissions) [de Oliveira et al., 2005]; 6, 3, 4, 2, and 2 for G. fulvus and G. rueppelli (12 fissions); 4, 4, 4, 2, and 2 for G. barbatus (11 fissions) [Nanda et al., 2006]; 5, 3, 4,2 , and 2 for L. albicollis (11 fissions) [de Oliveira et al., 2010]; and 7, 3, 4, 2, and 2 for N. n. orientalis (13 fissions) [present study], indicating that the fission patterns of chromosomes corresponding to GGA3-5 were identical among the 6 species. These results collectively suggest that a drastic karyotypic reorganization occurred early in the evolution of Accipitridae and that the accipitrid protokaryotype has been highly conserved in each lineage. A noteworthy characteristic of accipitrid karyotypes is the reduced chromosome number, although the frequent fragmentation of macrochromosomes should have increased the diploid number. A paint pool for 20 microchromosomes detected homology with 10 chromosomal segments and small chromosomes in N. n. orientalis, suggesting that fusion between microchromosomes and fusion of microchromosomes with larger chromosomes were the main causes of the decrease in chromosome number in this family; however, no signals of hybridization to telomeric repeats were found at interstitial fusion points. Drastic karyotypic reorganization has also been found in 3 species in Falconidae (common kestrel, F. tinnunculus; peregrine falcon, F. peregrinus; merlin, F. columbarius) [Nishida et al., 2008]. In these species, there was much less fission in ancestral macrochromosomes 1-5: the numbers of chromosome pairs that hybridized with GGA1-5 were 2, 2, 2, 2, and 2, respectively, for all 3 species. However, tandem fusions between microchromosomes and larger chromosomes occurred frequently as in accipitrid species. The stone curlew (Burhinus oedicnemus, Charadriiformes) also has a very low diploid chromosome number $(2 \mathrm{n}=42)$ with only 6 pairs of microchromosomes [Nie et al., 2009]. Reciprocal chromosome painting between chicken and this species revealed that this karyotype resulted from extensive microchromosome fusions, while macrochromosomal rearrangements hardly occurred except for a few 
fusions in smaller macrochromosomes corresponding to GGA6-9.

NORs have been found to be located predominantly on microchromosomes in birds [Sasaki et al., 1984, 1994; Schmid and Guttenbach, 1988], but in Accipitridae, NORs occur either on a single pair of macro- or microchromosomes or on 2 pairs of chromosomes [de Boer and Sinoo, 1984; de Lucca, 1985; Nishida-Umehara and Yoshida, 1994; Bed'Hom et al., 1998, 2003; Padilla et al., 1999; de Oliveira et al., 2005; Nishida et al., unpublished data]. The 18S-28S rRNA and MHC genes were localized to different pairs of microchromosomes in $N$. n. orientalis, whereas these genes are located close together on the same microchromosome in chicken (chromosome 16) and turkey [Groenen et al., 2000; Chaves et al., 2007; Delany et al., 2009], indicating that in one of the lineages an interchromosomal rearrangement occurred in the microchromosomes bearing the $18 \mathrm{~S}-28 \mathrm{~S}$ rRNA and MHC genes. In struthioniform species, the 18S-28S rRNA genes are also located on a single pair of microchromosomes [Gunski and Giannoni, 1998; Nishida-Umehara et al., 2007], but the chromosomal location of the MHC genes is not yet known. It thus remains unresolved, whether the intermicrochromosomal rearrangement occurred in the phasianid lineage or in N. n. orientalis.

Chicken microchromosomes, which are smaller than the 9 largest pairs of autosomal macrochromosomes and the ZW sex chromosomes, differ in several ways from macrochromosomes, not only in physical size but also in structure and function: high GC content, enriched CpG islands, high gene density, high acetylation of histone $\mathrm{H} 4$, low frequency of microsatellite DNA sequences, high meiotic recombination rates, etc. [McQueen et al., 1996, 1998; Primmer et al., 1997; Smith et al., 2000; International Chicken Genome Sequencing Consortium, 2004]. These results indicate that there is both structural and functional compartmentalization between macro- and microchromosomes in chicken. Microchromosome-specific centromeric repetitive sequences have been isolated from species in Galliformes and Struthioniformes [Matzke et al., 1990, 1992; Tanaka et al., 2000; Yamada et al., 2002a, b], which retain the ancestral state of the avian karyotype [Shetty et al., 1999; Kasai et al., 2003; Shibusawa et al., 2004a, b; NishidaUmehara et al., 2007]. The NNO-ApaI sequence isolated from $N$. n. orientalis is located in the centromeric heterochromatin of only 4 pairs of dot-shaped microchromosomes, and no homogenization of centromeric repetitive sequences has occurred between these microchromosomes and medium-sized or small chromo- somes. This result suggests that the chromosome sizedependent division of the genome was modified in N. $n$. orientalis as a consequence of drastic karyotype reorganization; however, genomic compartmentalization may remain in the 4 pairs of dot-shaped microchromosomes. There are 2 possible explanations for the chromosome size-dependent genomic compartmentalization in these microchromosomes. One is that they were not involved in chromosome rearrangements during the karyotype reorganization and, therefore, retain the primary structure of the ancestral microchromosomes. The alternative explanation, provided by the stone curlew (B. oedicnemus), is that the compartmentalization of microchromosomes was a result of genomic reorganization [Nie et al., 2009]. In the stone curlew, 4 dot-like microchromosomes (chromosomes 17-20) might have resulted from 3 fissions of a single ancestral microchromosome, opening the possibility that the amplification of centromeric repeated sequences and their homogenization are restricted to derivatives of the same microchromosome in N. n. orientalis. Further studies to analyze the molecular structures of these dot-shaped microchromosomes may shed more light on the origin of their genomic compartmentalization.

The NNO-ApaI family sequence has been conserved in Accipitridae, Pandionidae and Cathartidae, but not in Falconidae (F. peregrinus). Weak hybridization signals were also observed in 3 species of Gruiformes, suggesting that the NNO-ApaI sequence family was present in the common ancestor of Falconiformes and Gruiformes early in the evolution of Neoaves, was amplified in Accipitridae, Pandionidae and Cathartidae, but was not amplified or rapidly changed in Falconidae. Molecular cytogenetic characterization of centromeric repetitive sequences in other species will be required to better understand the process of genomic and karyotypic reorganization in accipitrids.

\section{Acknowledgements}

We thank Chiba Zoological Park for providing the specimens of common trumpeter; Kushiro Zoo for osprey; Nogeyama Zoological Gardens of Yokohama for Stanley crane and barn swallow; Okinawa Zoo and Museum Foundation for crested serpent eagle; Sapporo Maruyama Zoo for peregrine falcon; Tama Zoological Park for Siberian white crane, common coot, black-faced spoonbill, and northern goshawk; Ueno Zoological Gardens for Eurasian eagle owl and Andean condor; and Yokohama Zoological Gardens for emu. We obtained samples from Blakiston's fish owl and Japanese crested ibis with the cooperation of conservation programs organized by the Ministry of the Environment, Japan. We thank 
Dr. Hideyuki Tanabe (Graduate University for Advanced Studies, Japan) and Dr. Felix A. Habermann (Ludwig Maximilian University, Germany) for providing a mixture of chicken microchromosome paints and Prof. Matthew H. Dick (Hokkaido University, Japan) for valuable suggestions and critical reading of the manu- script. This work was supported by a Grant-in-Aid for Scientific Research (B) (No. 22370081) and a Grant-in-Aid for Scientific Research on Innovative Areas (No. 23113004) from the Ministry of Education, Culture, Sports, Science and Technology (MEXT), Japan.

\section{References}

Amaral KF, Jorge W: The chromosomes of the de Oliveira EHC, Tagliarini MM, Rissino JD, order Falconiformes: a review. Ararajuba 11: 65-73 (2003).

-Bed'Hom BT, Darré R, Fillon V: Chromosome banding studies in the Bateleur (Terathopius ecaudatus, Aves, Accipitridae). Chromosome Res 6:437-440 (1998).

Bed'Hom B, Coullin P, Guillier-Gencik Z, Moulin S, Bernheim A, Volobouev V: Characterization of the atypical karyotype of the blackwinged kite Elanus caeruleus (Falconiformes: Accipitridae) by means of classical and molecular cytogenetic techniques. Chromosome Res 11:335-343 (2003).

-Chaves LD, Krueth SB, Reed KM: Characterization of the turkey $\mathrm{MHC}$ chromosome through genetic and physical mapping. Cytogenet Genome Res 117:213-220 (2007).

Christidis L: Animal Cytogenetics 4: Chordata 3b: Aves (Gebrüder Borntraeger, Stuttgart 1990).

Crooijmans RP, Vrebalov J, Dijkhof RJ, van der Poel JJ, Groenen MA: Two-dimensional screening of the Wageningen chicken BAC library. Mamm Genome 11:360-363 (2000).

de Boer LEM: The somatic chromosome complements of 16 species of Falconiformes (Aves) and the karyological relationships of the order. Genetica 46:77-113 (1976).

de Boer LEM, Sinoo RP: A karyological study of Accipitridae (Aves: Falconiformes), with karyotypic descriptions of 16 species new to cytology. Genetica 65:89-107 (1984).

Delany ME, Robinson CM, Goto RM, Miller MM: Architecture and organization of chicken microchromosome 16: order of the NOR, $M H C$ $Y$, and $M H C-B$ subregions. J Hered 100:507514 (2009).

de Lucca EJ: Karyotype and nucleolus organizing regions in somatic chromosomes of the white-tailed hawk Buteo albicaudatus (Falconiformes: Aves). Cytobios 42:7-13 (1985).

$\checkmark$ de Oliveira EHC, Habermann FA, Lacerda O, Sbalqueiro IJ, Wienberg J, Müller S: Chromosome reshuffling in birds of prey: the karyotype of the world's largest eagle (harpy eagle, Harpia harpyja) compared to that of the chicken (Gallus gallus). Chromosoma 114: 338-343 (2005).

de Oliveira EHC, de Moura SP, dos Anjos LJS, Nagamachi CY, Pieczarka JC, et al: Comparative chromosome painting between chicken and spectacled owl (Pulsatrix perspicillata): implications for chromosomal evolution in the Strigidae (Aves, Strigiformes). Cytogenet Genome Res 122:157-162 (2008).
Pieczarka JC, Nagamachi CY, et al: Reciprocal chromosome painting between white hawk (Leucopternis albicollis) and chicken reveals extensive fusions and fissions during karyotype evolution of Accipitridae (Aves, Falconiformes). Chromosome Res 18:349-355 (2010).

Derjusheva S, Kurganova A, Habermann F, Gaginskaya E: High chromosome conservation detected by comparative chromosome painting in chicken, pigeon and passerine birds. Chromosome Res 12:715-723 (2004).

Griffin DK, Habermann F, Masabanda J, O’Brien P, Bagga M, et al: Micro- and macrochromosome paints generated by flow cytometry and microdissection: tools for mapping the chicken genome. Cytogenet Cell Genet 87:278-281 (1999).

Groenen MAM, Cheng HH, Bumstead N, Benkel $\mathrm{BF}$, Briles WE, et al: A consensus linkage map of the chicken genome. Genome Res 10:137147 (2000).

Gunski RJ, Giannoni ML: Nucleolar organizer regions and a new chromosome number for Rhea americana (Aves: Rheiformes). Genet Mol Biol 21:207-210 (1998).

Guttenbach M, Nanda I, Feichtinger W, Masabanda JS, Griffin DK, Schmid M: Comparative chromosome painting of chicken autosomal paints 1-9 in nine different bird species. Cytogenet Genome Res 103:173-184 (2003)

Habermann FA, Cremer M, Walter J, Kreth G, von Hase J, et al: Arrangements of macro- and microchromosomes in chicken cells. Chromosome Res 9:569-584 (2001).

Howell WM, Black DA: Controlled silver-staining of nucleolus organizer regions with a protective colloidal developer: a 1-step method. Experientia 36:1014-1015 (1980).

International Chicken Genome Sequencing Consortium (ICGSC): Sequence and comparative analysis of the chicken genome provide unique perspectives on vertebrate evolution.

Itoh Y, Arnold AP: Chromosomal polymorphism and comparative painting analysis in the zebra finch. Chromosome Res 13:47-56 (2005).

Kalitsis P, Choo KHA: Centromere DNA of higher eukaryotes, in Choo KHA (ed): The Centromere, pp 97-142 (Oxford University Press, New York 1997). Nature 432:695-716 (2004).
Kasai F, Garcia C, Arruga MV, Ferguson-Smith MA: Chromosome homology between chicken (Gallus gallus domesticus) and the red-legged partridge (Alectoris rufa); evidence of the occurrence of a neocentromere during evolution. Cytogenet Genome Res 102:326-330 (2003).

Masabanda JS, Burt DW, O’Brien PCM, Vignal A, Fillon V, et al: Molecular cytogenetic definition of the chicken genome: the first complete avian karyotype. Genetics 166:1367-1373 (2004)

Matsuda Y, Chapman VM: Application of fluorescence in situ hybridization in genome analysis of the mouse. Electrophoresis 16: 261-272 (1995).

Matsuda Y, Nishida-Umehara C, Tarui H, Kuroiwa A, Yamada K, et al: Highly conserved linkage homology between birds and turtles: birds and turtle chromosomes are precise counterparts of each other. Chromosome Res 13:601615 (2005).

Matzke MA, Varga F, Berger H, Schernthaner J, Schweizer D, et al: A 41-42 bp tandemly repeated sequence isolated from nuclear envelopes of chicken erythrocytes is located predominantly on microchromosomes. Chromosoma 99:131-137 (1990).

Matzke AJM, Varga F, Gruendler P, Unfried I, Berger $\mathrm{H}$, et al: Characterization of a new repetitive sequence that is enriched on microchromosomes of turkey. Chromosoma 102: 9-14 (1992).

McQueen HA, Fantes J, Cross SH, Clark VH, Archibald AL, Bird AP: CpG islands of chicken are concentrated on microchromosomes. Nat Genet 12:321-324 (1996).

-McQueen HA, Siriaco G, Bird AP: Chicken microchromosomes are hyperacetylated, early replicating, and gene rich. Genome Res 8: 621-630 (1998).

Nanda I, Karl E, Volobouev V, Griffin DK, Schartl M, Schmid M: Extensive gross genomic rearrangements between chicken and Old World vultures (Falconiformes: Accipitridae). Cytogenet Genome Res 112:286-295 (2006).

Nanda I, Karl E, Griffin DK, Schartl M, Schmid $\mathrm{M}$ : Chromosome repatterning in three representative parrots (Psittaciformes) inferred from comparative chromosome painting. $\mathrm{Cy}$ togenet Genome Res 117:43-53 (2007).

Nanda I, Schlegelmilch K, Haaf T, Schartl M, Schmid M: Synteny conservation of the Z chromosome in 14 avian species ( 11 families) supports a role for $\mathrm{Z}$ dosage in avian sex determination. Cytogenet Genome Res 122: 150-156 (2008). 
Nanda I, Benisch P, Fetting D, Haaf T, Schmid M: Synteny conservation of chicken macrochromosomes 1-10 in different avian lineages revealed by cross-species chromosome painting. Cytogenet Genome Res 132:165-181 (2011).

-Nie W, O’Brien PCM, Ng BL, Fu B, Volobouev V, et al: Avian comparative genomics: reciprocal chromosome painting between domestic chicken (Gallus gallus) and the stone curlew (Burhinus oedicnemus, Charadriiformes) - an atypical species with low diploid number. Chromosome Res 17:99-113 (2009).

- Nishida C, Ishijima J, Kosaka A, Tanabe H, Habermann FA, et al: Characterization of chromosome structures of Falconinae (Falconidae, Falconiformes, Aves) by chromosome painting and delineation of chromosome rearrangements during their differentiation. Chromosome Res 16:171-181 (2008).

Nishida-Umehara C, Yoshida MC: The karyotypes of nine golden eagles, Aquila chrysaetos. Chrom Inform Serv 56:22-24 (1994).

- Nishida-Umehara C, Tsuda Y, Ishijima J, Ando J, Fujiwara A, et al: The molecular basis of chromosome orthologies and sex chromosomal differentiation in palaeognathous birds. Chromosome Res 15:721-734 (2007).

- Padilla JA, Martinez-Trancón M, Rabasco A, Fernández-García JL: The karyotype of the Iberian imperial eagle (Aquila adalberti) analyzed by classical and DNA replication banding. Cytogenet Cell Genet 84:61-66 (1999).

- Primmer CR, Raudsepp T, Chowdhary BP, Moller AP, Ellegren H: Low frequency of microsatellites in the avian genome. Genome Res 7:471-482 (1997).
Raudsepp T, Houck ML, O'Brien PC, FergusonSmith MA, Ryder OA, Chowdhary BP: Cytogenetic analysis of California condor (Gymnogyps californianus) chromosomes: comparison with chicken (Gallus gallus) macrochromosomes. Cytogenet Genome Res 98: 54-60 (2002).

Sasaki M, Takagi N, Nishida C: Current profiles of avian cytogenetics, with notes on chromosomal diagnosis of sex in birds. the nucleus 27: 63-73 (1984).

Sasaki M, Nishida-Umehara C, Tsuchiya K: Interspecific variations in centromeric C-band of the $\mathrm{Z}$ chromosome and silver stained nucleolus organizer regions (Ag-NORs) among ten species of owls (Strigiformes). Chromosome Inf Serv 56:19-21 (1994).

Schmid M, Guttenbach M: Evolutionary diversity of reverse (R) fluorescent chromosome bands in vertebrates. Chromosoma 97:101-114 (1988).

Shetty S, Griffin DK, Graves JAM: Comparative painting reveals strong chromosome homology over 80 million years of bird evolution. Chromosome Res 7:289-295 (1999).

-Shibusawa M, Nishibori M, Nishida-Umehara C, Tsudzuki M, Masabanda J, et al: Karyotypic evolution in the Galliformes: an examination of the process of karyotypic evolution by comparison of the molecular cytogenetic findings with the molecular phylogeny. Cytogenet Genome Res 106:111-119 (2004a).

Shibusawa M, Nishida-Umehara C, Tsudzuki M, Masabanda J, Griffin DK, Matsuda Y: A comparative karyological study of the blue-breasted quail (Coturnix chinensis, Phasianidae) and California quail (Callipepla californica, Odontophoridae). Cytogenet Genome Res 106:82-90 (2004b).

-Smith J, Bruley CK, Paton IR, Dunn I, Jones CT, et al: Differences in gene density on chicken macrochromosomes and microchromosomes. Anim Genet 31:96-103 (2000).
Sumner AT: A simple technique for demonstrating centromeric heterochromatin. Exp Cell Res 75:304-306 (1972).

Takagi N, Sasaki M: A phylogenetic study of bird karyotypes. Chromosoma 46:91-120 (1974).

Tanaka K, Suzuki T, Nojiri T, Yamagata T, Namikawa T, Matsuda Y: Characterization and chromosomal distribution of a novel satellite DNA sequence of Japanese quail (Coturnix coturnix japonica). J Hered 91:412-415 (2000).

Uno Y, Nishida C, Tarui H, Ishishita S, Takagi C, et al: Inference of the protokaryotypes of amniotes and tetrapods and the evolutionary processes of microchromosomes from comparative gene mapping. PLoS One 7:e53027 (2012).

- Yamada K, Nishida-Umehara C, Matsuda Y: Characterization and chromosomal distribution of novel satellite DNA sequences of the lesser rhea (Pterocnemia pennata) and the greater rhea (Rhea americana). Chromosome Res 10:513-523 (2002a).

-Yamada K, Shibusawa M, Tsudzuki M, Matsuda Y: Molecular cloning and characterization of novel centromeric repetitive DNA sequences in the blue-breasted quail (Coturnix chinensis, Galliformes). Cytogenet Genome Res 98:255261 (2002b).

-Yamada K, Nishida-Umehara C, Matsuda Y: A new family of satellite DNA sequences as a major component of centromeric heterochromatin in owls (Strigiformes). Chromosoma 112:277-287 (2004).

-Yamada K, Nishida-Umehara C, Ishijima J, Murakami $\mathrm{T}$, Shibusawa $\mathrm{M}$, et al: A novel family of repetitive DNA sequences amplified sitespecifically on the $\mathrm{W}$ chromosomes in neognathous birds. Chromosome Res 14:613627 (2006). 